\title{
Coverage Probability Analysis by Fractional Frequency Reuse Scheme
}

\author{
Joydev Ghosh ${ }^{1}$, Dushanta Nalin K. Jayakody ${ }^{2,1}$, Marwa Qaraqe ${ }^{2}$, and \\ Theodoros A. Tsiftsis ${ }^{3}$ \\ 1 School of Computer Science and Robotics, National Research Tomsk Polytechnic \\ University, RUSSIA, \\ 2 College of Science and Engineering Hamad Bin Khalifa University, Qatar \\ Foundation, Education City, Doha, QATAR \\ 3 School of Engineering, Nazarbayev University, KAZAKHASTAN \\ E-mail:joydev.ghosh.ece@gmail.com, nalin.jayakody@ieee.org, \\ mqaraqe@hbku.edu.qa and theodoros.tsiftsis@nu.edu.kz
}

\begin{abstract}
In this work, we present the cell edge coverage probability (CECP) performance of cellular networks under the composite multipath fading environment, where Rayleigh fading is superimposed on lognormal shadowing, by the fractional frequency reuse (FFR) scheme. We demonstrate that our analytical results and the simulation results are in line with the analysis presented in [12].
\end{abstract}

Keywords: Cell Edge Coverage Probability (CECP), Fractional Frequency Reuse (FFR) Scheme, Composite Multi-path Fading

\section{Introduction}

Drastically growing energy costs and rising rigid scenario standards have led to an increased demand of addressing the energy efficiency (EE) aspect of next generation networks [1]. In early wireless networks, the radio access portion consumes more than $2 / 3$ of the total energy consumption [2]. Hence, improving the energy efficiency (EE) of wireless networks is very important in order to fulfill the constraint issues that arise by the large requirement of traffic and energy consumption. The femtocell is an emerging and non-discriminatory issue in current and next generation radio access networks [3]. Owing to its small transmission path between transmitter and receiver and low transmit power feature, the femtocell can reduce the consumption of energy, increase the battery life of low-power smart devices, and increase the network coverage in terms of the quality of experience (QoE) [4]. As of today, a great deal of work has been dedicated to resource distribution and interference reduction techniques in future generation networks with small cell. The majority of the work articulate on the orthogonal frequency division multiple access (OFDMA) resource allocations [5] and sub-channel allocations [6] to reduce the cross tier interference. The fractional frequency reuse (FFR) scheme is considered as one of the efficient 
interference mitigation techniques in cellular networks. This can reduces crosstier interference and optimize spatial reuse. In the the conventional approach, where the frequency reuse factor $(F R F)=1$, the entire frequencies to each macrocell provides large spatial reuse, but less coverage. In the this approach, where the $F R F=3,(1 / 3)$ of whole spectrum resources in individual macrocell can provide a less spatial reuse, but large coverage. We treat coverage probability (CP) as our performance metric. This can be defined as the probability that the achieved signal to interference plus noise ratio (SINR) higher than the required threshold for better quality of experience (QoE) [7]. Coverage probability is an important parameter to compute the communication reliability. Below, we outline the contribution given in this work.

1. We analytically derive the expressions for the CECP metric of a FFR scheme by explicitly considering the independent composite multi-path fading power for inner cell users (ICUs) and outer cell users (OCUs). We extend the analysis to obtain an expression for the $\mathrm{CP}$ metric of this network.

2. We show that the analytical results for the CECP converge to the corresponding simulation for all values of path loss exponents, $\alpha$.

\section{System Model}

We consider OFDMA based multi-cellular DL wireless networks where the macro base stations (MBSs) are distributed as a spatial Poisson point process (PPP) and users are distributed uniformly [8]. For the sake of simplicity of analysis, we assume $k$ number of interfering MBSs with density $\rho$. The SINR of a user associated to the macrocell centre can be expressed as [9]:

$$
\gamma_{c c}=\frac{P_{0} h_{0}\left\|d_{0}\right\|^{-\alpha}}{\sum_{j \in k} P_{j} \tau_{j} h_{j}\left\|D_{j}\right\|^{-\alpha}+N_{0}},
$$

where $P_{0}$ indicates transmit power by the reference user located at random position $\left(x_{0}, y_{0}\right), h_{0}$ and $\alpha$ indicates the composite multi-path fading and path loss exponent, respectively. The probability density function (PDF) of the SINR can be expressed as in [10]

$$
p_{\gamma}(\gamma)=\int_{0}^{\infty} \frac{1}{\bar{\gamma} \Gamma(1)} \exp \left(-\frac{\gamma}{\bar{\gamma}}\right) \frac{\xi}{\sqrt{2 \pi} \sigma \gamma} \exp \left[-\frac{\left(10 \log _{10} \gamma-\mu\right)^{2}}{2 \sigma^{2}}\right] d \gamma,
$$

where $\bar{\gamma}$ denotes the average SINR per symbol, $\gamma$ is distributed according to the gamma distribution $\Gamma(1)=\int_{0}^{\infty} e^{-t} d t, \xi=10 / \ln 10=4.343, \mu(\mathrm{dB})$, and $\sigma(\mathrm{dB})$ are the mean and standard deviation of $10 \log _{10} \gamma$ respectively. $P_{j} \tau_{j} h_{j}\left\|D_{j}\right\|^{-\alpha}$, $\forall j=0,1,2, \ldots, J$ stands for the received power from a base station (BS) positioned at $\left(x_{j}, y_{j}\right) .\left\|D_{j}\right\|=R \sqrt{3} \sqrt{\left(x_{j}-\phi x_{0}\right)^{2}+\left(y_{j}-\phi y_{0}\right)^{2}+\left(x_{j}-\phi x_{0}\right)\left(y_{j}-\phi y_{0}\right)}$ indicates $j^{t h}$ BS and the user positioned at $\left(x_{0}, y_{0}\right)$ where $\phi=\frac{R_{t}}{R} \in[0,1]$ indicates dividing condition between middle and edge of the macrocell. $\tau_{j}=0,1$ 


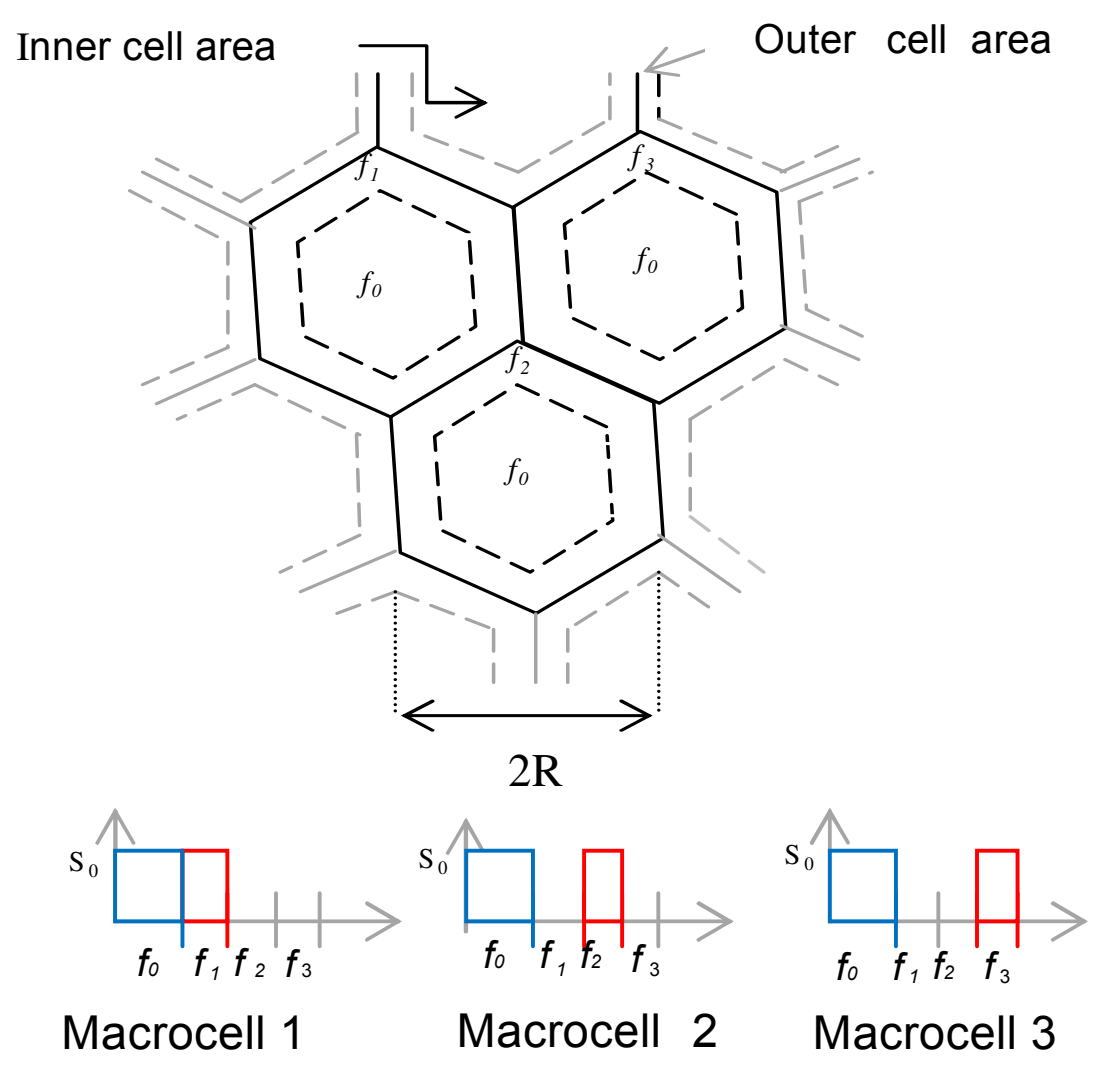

Fig. 1. FFR with three macrocell clusters in size

represents an indicator parameter to find the status, i.e, inactive or busy, of each channel and $N_{0}$ is the additive white Gaussian noise (AWGN) power [11].

\subsection{Cell Edge Coverage Probability (CECP):}

In this subsection, we obtain the CECP analytically using FFR scheme. The CECP has already been analyzed in [12]. Also, it converges to the simulation outcomes in [12]. The CECP of a mobile user at a separation $d_{0}$ from the MBS in FFR scheme can be expressed as $P_{c e}\left(d_{0}\right)_{\mid(s)}=\left[\gamma_{c e}\left(d_{0}\right)>\gamma_{t r g} \mid \gamma_{c c}\left(d_{0}\right)<\gamma_{t h}\right]$ . Thus we have 


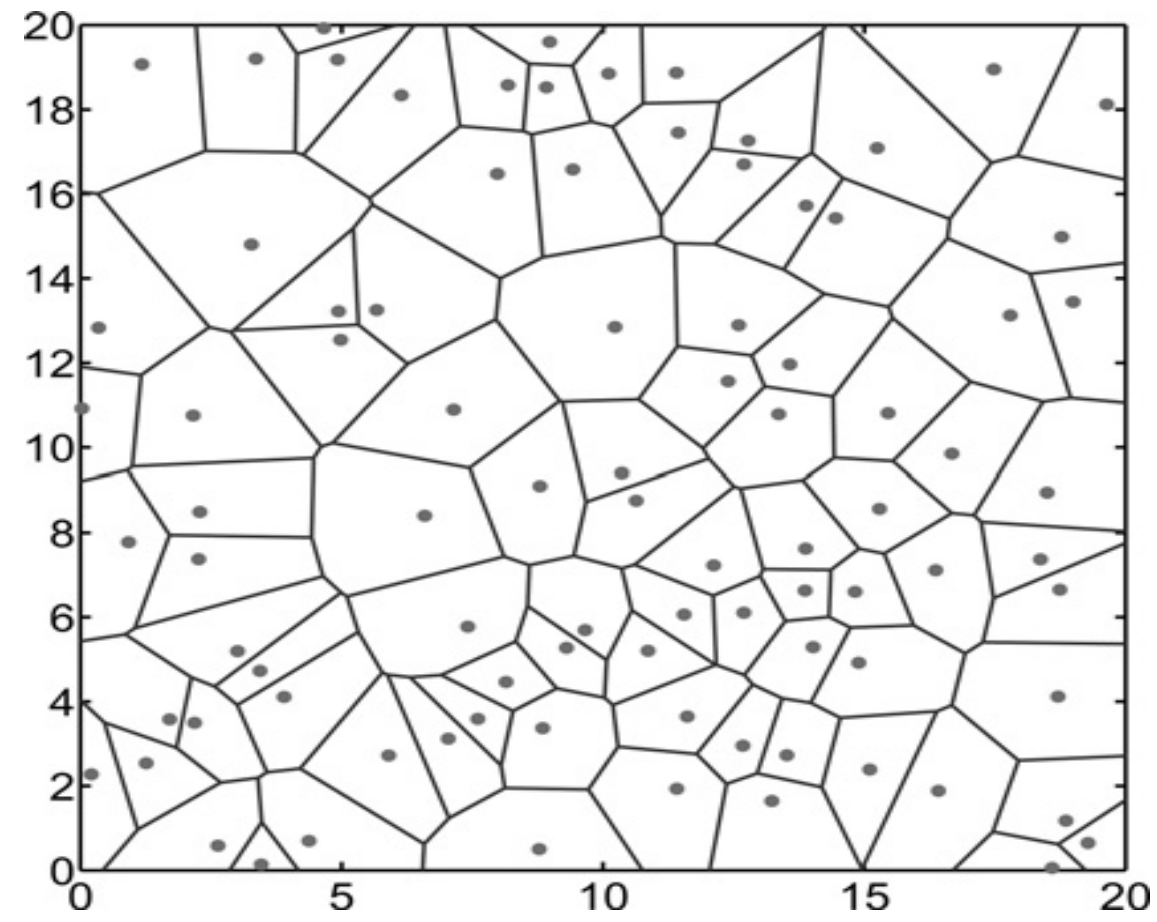

Fig. 2. FBSs are distribute as spatial PPP and femtocell boundaries conform to a Voronoi tessellation

$P_{c e}\left(d_{0}\right)=P\left[\frac{P_{0} \overline{h_{0}}\left\|d_{0}\right\|^{-\alpha}}{\sum_{j \in m} P_{j} \tau_{j} \overline{h_{j}}\left\|D_{j}\right\|^{-\alpha}+N_{0}}>\gamma_{t r g} \mid \frac{P_{0} h_{0}\left\|d_{0}\right\|^{-\alpha}}{\sum_{j \in m} P_{j} \tau_{j} h_{j}\left\|D_{j}\right\|^{-\alpha}+N_{0}}<\gamma_{t h}\right]$

where, $\gamma_{c e}\left(d_{0}\right)$ and $\gamma_{c c}\left(d_{0}\right)$ indicate the SINR of a user located at outer cell area $(\mathrm{OCA})$ and inner cell area (ICA), respectively, $\overline{h_{0}}$ and $\overline{h_{j}}$ indicate composite multi-path fading experienced by the users located at OCA, $m$ is the number of interfering MBS at cell edge, $\gamma_{t r g}$ and $\gamma_{t h}$ indicate SINR threshold and target SINR, respectively. Here, $(s)$ signifies that the SINR of a user located at cell edge is less than $\gamma_{t h}$, i.e., any user whose SINR is lower than $\gamma_{t h}$ is to be considered as a cell edge user. Then allocates a resource to them by FFR scheme. By considering that the channel fading power is independent and identically distributed random variables between the sub-bands, we get

$$
P_{c e}\left(d_{0}\right)=P\left[\gamma_{c e}\left(d_{0}\right)>\gamma_{t r g} \mid \gamma_{c c}\left(d_{0}\right)<\gamma_{t h}\right]=P\left[\gamma_{c e}\left(d_{0}\right)>\gamma_{t r g}\right]
$$


The CECP of a typical user can be expressed as:

$$
P_{c e}=\frac{\left.\int_{0}^{\infty} P\left[\gamma_{c e}\left(d_{0}\right)>\gamma_{t r g} \mid \gamma_{c c}\left(d_{0}\right)<\gamma_{t h}\right] P\left[\gamma_{c c}\left(d_{0}\right)<\gamma_{t h}\right] p_{\gamma}\left(d_{0}\right) d d_{0}\right)}{\int_{0}^{\infty}\left[\gamma_{c c}\left(d_{0}\right)<\gamma_{t h}\right] p_{\gamma}\left(d_{0}\right) d d_{0}},
$$

where, $p_{\gamma}\left(d_{0}\right)$ indicates the probability density function (PDF) of distance, $d_{0}$, between cell user and nearest FBS, and it can be expressed as $p_{\gamma}\left(d_{0}\right)=$ $2 \pi \rho d_{0} e^{-\rho \pi d_{0}^{2}}, d_{0}>0$ [13]. Substituting (4) in (5), we get:

$$
P_{c e}=\frac{\left.\int_{0}^{\infty} P\left[\gamma_{c e}\left(d_{0}\right)>\gamma_{t r g}\right] P\left[\gamma_{c c}\left(d_{0}\right)<\gamma_{t h}\right] p_{\gamma}\left(d_{0}\right) d d_{0}\right)}{\int_{0}^{\infty}\left[\gamma_{c c}\left(d_{0}\right)<\gamma_{t h}\right] p_{\gamma}\left(d_{0}\right) d d_{0}},
$$

In [13], it has already been expressed:

$$
\begin{aligned}
& P\left[\gamma_{c c}\left(d_{0}\right)>\gamma_{t r g}\right]=\exp \left[-\pi \rho d_{0}^{2} p\left(\gamma_{t r g}, \alpha, \delta_{I C A}\right)-\gamma_{t r g} N_{0} d_{0}^{\alpha}\right], \\
& P\left[\gamma_{c c}\left(d_{0}\right)>\gamma_{t r g}\right]=\exp \left[-\pi \rho d_{0}^{2} p\left(\gamma_{t r g}, \alpha, \delta_{O C A}\right)-\gamma_{t r g} N_{0} d_{0}^{\alpha}\right],
\end{aligned}
$$

where, an FRF assigned to the users located at inner cell area (ICA) is denoted by $\delta_{I C A}$ whereas $\delta_{O C A}$ is considered for the users located at outer cell area (OCA), where $\delta_{I C A}<\delta_{O C A}$.

$$
\begin{aligned}
p\left(\gamma_{t r g}, \alpha, \delta_{O C A}\right) & =\gamma_{t r g}^{2} \alpha \int_{\gamma_{\operatorname{trg}}^{-\frac{2}{\alpha}}}^{\infty} \frac{1}{u^{\frac{\alpha}{2}}+1} d u \\
& =\frac{2 \gamma_{\operatorname{trg}}}{\delta_{O C A}(\alpha-2)}{ }_{2} F_{1}\left(1,(\alpha-2) / \alpha, 2-2 / \alpha,-\gamma_{t r g}\right),
\end{aligned}
$$

where, ${ }_{2} F_{1}\left(1,(\alpha-2) / \alpha, 2-2 / \alpha,-\gamma_{\text {trg }}\right)$ denotes the Gauss hypergeometric function [14]. Hence, $P_{c e}$ can be further re-expressed as:

$$
P_{c e}=\frac{\pi \rho \int_{0}^{\infty} e^{-\pi p d_{0}} e^{-\pi p d_{0} p\left(\gamma_{t r g}, \alpha, \delta_{O C A}\right)-\gamma_{t r g} N_{0} d_{0}^{\frac{\alpha}{2}}}\left(1-e^{-\pi p d_{0} p\left(\gamma_{t h}, \alpha, \delta_{I C A}\right)-\gamma_{t h} N_{0} d_{0}^{\frac{\alpha}{2}}}\right) d d_{0}}{1-\pi \rho \int_{0}^{\infty} e^{-\pi p d_{0}\left(1+p\left(\gamma_{t h}, \alpha, \delta_{I C A}\right)\right)-\gamma_{t h} N_{0} d_{0}^{\frac{\alpha}{2}}} d d_{0}} .
$$

The respective derivation in [12] for CECP of a user in a FFR scheme can be expressed as:

$$
\begin{gathered}
P_{c e,[12]}=\frac{\pi \rho \int_{0}^{\infty} e^{-\pi p d_{0}\left(1+p\left(\gamma_{t r g}, \alpha, \delta_{O C A}\right)\right)-\gamma_{t r g} N_{0} d_{0}^{\frac{\alpha}{2}}} d d_{0}}{1-\pi \rho \int_{0}^{\infty} e^{-\pi p d_{0}\left(1+p\left(\gamma_{t h}, \alpha, \delta_{I C A}\right)\right)-\gamma_{t h} N_{0} d_{0}^{\frac{\alpha}{2}}} d d_{0}} \\
-\frac{\pi \rho \int_{0}^{\infty} e^{-\pi p d_{0}} e^{-\pi p d_{0} 2 \xi\left(\gamma_{t r g}, \gamma_{t h}, \alpha, \delta_{O C A}\right)-\gamma_{t r g} N_{0} d_{0}^{\frac{\alpha}{2}}} d d_{0}}{1-\pi \rho \int_{0}^{\infty} e^{-\pi p d_{0}\left(1+p\left(\gamma_{t h}, \alpha, \delta_{I C A}\right)\right)-\gamma_{t h} N_{0} d_{0}^{\frac{\alpha}{2}}} d d_{0}}
\end{gathered}
$$

where, $\xi\left(\gamma_{t r g}, \gamma_{t h}, \alpha, \delta_{O C A}\right)$

$=\int_{1}^{\alpha}\left[1-\frac{1}{1+\gamma_{t h} r^{-\alpha}}\left(1-\frac{1}{\delta_{O C A}}\left(1-\frac{1}{1+\gamma_{t r g} r^{-\alpha}}\right)\right)\right] d r$. In the noise free scenario, we can again re-write $P_{c e}$ by solving integrals of (10) as: 


$$
P_{c e}=\frac{1+p\left(\gamma_{t h}, \alpha, \delta_{O C A}\right)}{\left(1+p\left(\gamma_{t r g}, \alpha, \delta_{O C A}\right)\right)\left(1+p\left(\gamma_{t r g}, \alpha, \delta_{O C A}\right)+p\left(\gamma_{t h}, \alpha, \delta_{I C A}\right)\right)}
$$

In the noise free scenario, the respective expressions in [12] can be re-written as:

$$
P_{(c e,[12])}=\frac{1+p\left(\gamma_{t h}, \alpha, \delta_{I C A}\right)}{p\left(\gamma_{t h}, \alpha, \delta_{I C A}\right)}\left(\frac{1}{p\left(\gamma_{t r g}, \alpha, \delta_{O C A}\right)}-\frac{1}{1+2 \xi\left(\gamma_{t r g}, \gamma_{t h}, \alpha, \delta_{O C A}\right)}\right)
$$

At $\alpha=4, P_{c e}$ can be again expressed by:

$$
P_{c e}=\frac{1+\sqrt{\gamma_{t h}} \arctan \left(\gamma_{t h}\right)}{\left(1+\left(\frac{1}{\delta}\right) \sqrt{\gamma_{\text {trg }}} \arctan \left(\gamma_{t r g}\right)\right)\left(1+\left(\frac{1}{\delta}\right)\right) \sqrt{\gamma_{t r g}} \arctan \left(\gamma_{t r g}\right)+\sqrt{\gamma_{t h}} \arctan \left(\gamma_{t h}\right)}
$$

Further, the respective expression in [12] can be written by:

$$
\begin{aligned}
P_{(c e,[12])}= & \frac{1+\sqrt{\gamma_{t h}} \arctan \left(\gamma_{t h}\right)}{\sqrt{\gamma_{t h}} \arctan \left(\gamma_{t h}\right)} \times \\
& \left(\frac{1}{1+\left(\frac{1}{\delta}\right) \sqrt{\gamma_{t r g}} \arctan \left(\gamma_{t r g}\right)}-\frac{1}{1+2 \xi\left(\gamma_{t r g}, \gamma_{t h}, 4, \delta_{O C A}\right)}\right) .
\end{aligned}
$$

To find the coverage probability $(\mathrm{CP})$ of the FFR scheme, we initially denote CP of a particular CU by $P_{c p}$. Thus,

$$
P_{c p}=\int_{d_{0}>0}\left(P_{c c} P\left[\gamma_{c e}\left(d_{0}\right)>\gamma_{t h}\right]+P_{c e} P\left[\gamma_{c e}\left(d_{0}\right)<\gamma_{t h}\right]\right) p_{\gamma}\left(d_{0}\right) d d_{0},
$$

where, the term $P_{c c} P\left[\gamma_{c e}\left(d_{0}\right)>\gamma_{t h}\right]$ stands for the CP due to ICUs and the another term $P_{c e} P\left[\gamma_{c e}\left(d_{0}\right)<\gamma_{t h}\right]$ stands for the CP due to OCUs. The CP at cell centre can be written as in [12, Theorem 2]:

$$
P_{c c}=\frac{P\left[\gamma_{c e}\left(d_{0}\right)>\max \left(\gamma_{t r g}, \gamma_{t h}\right)\right]}{P\left[\gamma_{c e}\left(d_{0}\right)>\gamma_{t h}\right]} .
$$

Applying (12) and (17), $P_{c p}$ can be further simplified as:

$$
\begin{aligned}
P_{c p}=\frac{1}{1+p\left(\max \left(\gamma_{t r g}, \gamma_{t h}\right), \alpha, \delta_{I C A}\right)} & +\frac{1}{1+p\left(\gamma_{t r g}, \alpha, \delta_{O C A}\right)} \\
& -\frac{1}{p\left(\gamma_{t h}, \alpha, \delta_{I C A}\right)+p\left(\gamma_{t r g}, \alpha, \delta_{O C A}\right)} .
\end{aligned}
$$

\section{Results and discussion}

It can be seen that the CECP expressions in (10), (12) and (14) are computationally easy to compute as compared to corresponding expressions in (11), 


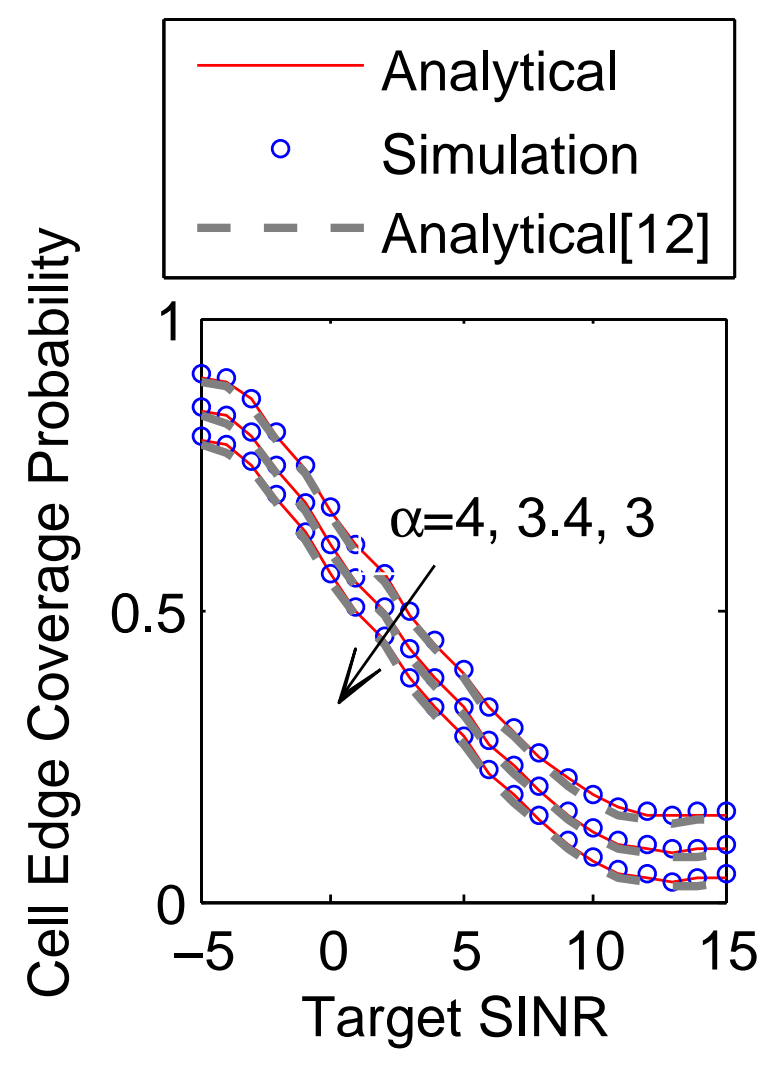

Fig. 3. Contrast of our analytical results, results provided in [12] and simulations for $\alpha=3,3.4,4$, where, $\delta_{O C A}=3, \gamma_{t h}=1 \mathrm{~dB}, \rho=0.3 \mathrm{Km}^{2}$

(13) and (15), successively. Thereafter, we contrast the analytical outcomes to the simulation outcomes. For the purpose of computations, we choose a $20 \mathrm{Km}$ x $20 \mathrm{Km}$ surface area and produce the arbitrary positions of MBSs applying spatial PPP of density $\rho$. As illustrated in Fig. 2, cell users (CUs) have scattered uniformly in this specified area and individual CU is served by the close FBS and femtocell boundaries conform to a Voronoi tessellation. We consider inner cell and for individual CU belongs to inner cell, we induce composite multipath fading belonging to its own transmission link and composite multipath fading belonging to the rest FBSs, which is unwanted interference power and compute SINR per CU. Further, we categorize the entire users into outer cell users (OCUs) and inner cell users (ICUs) depending upon the preset SINR threshold. Then, for the OCUs, we further induce composite multipath fading belonging to its own transmission link and composite multipath fading belonging to the outer region of the rest FBSs as multipath fading is independent between OCUs and ICUs. Then, we again compute the new SINR and compare the new SINR of each user with $\gamma_{t r g}$, and iterate this 20,000 times to achieve the CECP. Thereafter, we 


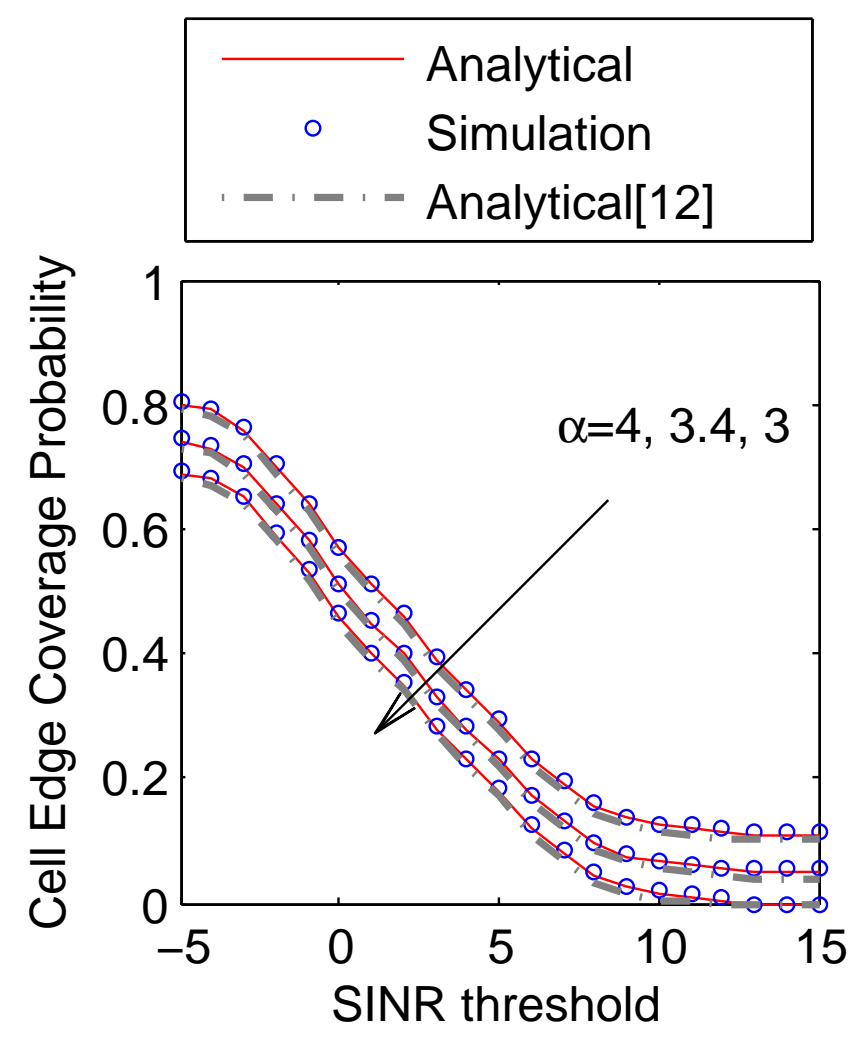

Fig. 4. Contrast of our analytical results, results provided in [12] and simulations for a range of $\gamma_{t h}$, where, $\delta_{O C A}=3, \gamma_{t h}=\gamma_{t r g}, \rho=0.3 \mathrm{Km}^{2}$

take on spectrum assignment in FFR scheme, where the entire spectrum and spectrum assigned to the ICUs are denoted by $f$ and $f_{0}$. Thus, as in [12], we get, $\frac{f_{0}}{f}=\int_{d_{0}=0}^{\infty} P\left[\gamma\left(d_{0}\right)>\gamma_{t r g} p_{\gamma}\left(d_{0}\right) d d_{0}\right]$. Besides, $f_{1}=f_{2}=f_{3}=\frac{\left(f-f_{0}\right)}{3}$, where $f_{1}, f_{2}, f_{3}$ are spectrum allocated to the OCUs for different macrocells.

Fig. 3 differentiates our contributions obtained from the analysis, the results presented in the analysis of [12], and the simulations results for $\alpha=3,3.4,4$, respectively. Furthermore, in Fig. 4, both the outcomes achieved from the analysis and the simulations are contrasted for the variation of $\gamma_{t h}$. This can be found that our contributions obtained from the analysis, the outcomes obtained from the analysis in [12], and the outcomes from the simulations are entirely in so close match, in both Fig. 3 and Fig. 4. Moreover, our contributions obtained from the analysis are comparatively closer to the simulations results for all values of $\alpha$. 


\section{Conclusion}

We have presented the numerical expressions of the CECP metric for FFR scheme and then successfully demonstrated through the simulation to demonstrate that the accuracy of our analytical work. We conclude that both our contributions and the analytical results in [12] are exactly converged to the simulation results.

\section{References}

1. Yu, F. R., Zhang, X., and Leung, V. C. M.: Green Communications and Networking. New York: CRC Press (2012).

2. Chen, Y., Zhang, S., Xu, S., and Li, G. Y.: Fundamental trade-offs on green wireless networks. IEEE Comm. Mag., vol. 49, no. 6, pp. 3037 (2011).

3. Chandrasekhar, V., Andrews, J., and Gatherer, A.: Femtocell networks: a survey. IEEE Communications Magazine, 46(9), 5967 (2008).

4. Ghosh, J., and Roy, S. D.: Qualitative analysis for coverage probability and energy efficiency in cognitive-femtocell networks under macrocell infrastructure. Electronics Letters, 51(17), (2015).

5. Liang, Y., Chung, W., Ni, G., Chen, I., Zhang, H., and Kuo, S.: Resource allocation with interference avoidance in OFDMA femtocell networks. IEEE Transactions on Vehicular Technology. 61(5):22432255 (2012).

6. Cheung, W., Quek, T., and Kountouris, M.: Throughput optimization, spectrum allocation, and access control in two-tier femtocell networks. IEEE Journal on Selected Areas in Communications. 30(3):561574 (2012).

7. Yang, X., and Fapojuwo, A. O.: Coverage Probability Analysis of Heterogeneous Cellular Networks in Rician/Rayleigh Fading Environments. IEEE Communications Letters, 19(7), pp.1197-1200 (2015).

8. Chinnadurai, S., Selvaprabhu, P., Jeong, Y., Sarker, A. L., Hai, H., Duan, W., and Lee, M. H.: User clustering and robust beamforming design in multicell MIMONOMA system for 5G communications. AEU - International Journal of Electronics and Communications, Volume 78, Pages 181191 (2017).

9. Ghosh, J., Jayakody, D.N.K, and Qaraqe, M.:Cognitive-Femtocell Based Resource Allocation in Macrocell Network. 28th Annual IEEE International Symposium on Personal, Indoor and Mobile Radio Communications (PIMRC),Montreal, Canada,2017.

10. Hansen, F., and Meno., F. I.: Mobile fading-Rayleigh and log normal superimposed. IEEE Transactions on Vehicular Technology., vol. VT-26, pp. 332335 (1977).

11. Simon, M. K., and Alouini, M. S.: Fading Channel Characterization and Modeling. Digital Communication Over Fading Channels, (2002).

12. Novlan, T. D., Ganti, R. K., Ghosh, A., and Andrews, J. G.: Analytical evaluation of fractional frequency reuses for OFDMA cellular networks. IEEE Trans. Wireless Commun., 10(12), pp. 42944305 (2011).

13. Andrews, J. G., Baccelli, F., Ganti, R. K.: A tractable approach to coverage and rate in cellular networks. IEEE Trans. Commun., 59(11), pp. 31223134 (2011).

14. Gradshteyn, I. S., Ryzhik, I. M.: Table of integrals, series, and products. Elsevier/Academic Press,7th edn. (2007). 